hep-th/9307161

IHEP 92-146

October, 1992

\title{
THE CANONICAL SYMMETRY FOR INTEGRABLE SYSTEMS
}

\author{
A. N. Leznov and A. V. Razumov' \\ Institute for High Energy Physics, 142284 Protvino, Moscow Region, Russia
}

\begin{abstract}
The properties of discrete nonlinear symmetries of integrable equations are investigated. These symmetries are shown to be canonical transformations. On the basis of the considered examples, it is concluded, that the densities of the conservation laws are changed under these transformations by spatial divergencies.
\end{abstract}

\footnotetext{
${ }^{1}$ E-mail: razumov@mx.ihep.su
} 


\section{Introduction}

Recently the discrete nonlinear symmetry transformations for the variety of the integrable systems were obtained. [1, 2, 3] These transformations were given in an explicit form, which allows one to get the whole hierarchy of solutions from any initial solution of the given nonlinear equation. In particular, the soliton solutions can be obtained with the help of these transformations without using the inverse scattering method. Unfortunately, we do not know a constructive method to find the corresponding discrete symmetry transformation for an arbitrary integrable systems. Actually such transformations arose as a byproduct of the algebraic approach to the construction of the soliton solutions of integrable systems. [4

In this paper we investigate the properties of the discrete symmetry from the point of view of the Hamiltonian structure relevant to integrable systems. Numerous examples, not only two dimensional ones, lead us to the conclusion that all these transformations are canonical.

For the case of the nonlinear Schrödinger equation, the densities of the lowest conservation laws appeared to be shifted under these transformations by total space derivatives. It can be shown that all the densities of the conservation laws possess this property, and, in a sense, these densities are invariants of the transformations under consideration. Because of the importance of this property we shall devote to its proof a separate paper.

Note that the symmetry transformations, considered in the present paper, are very different from the well known discrete symmetry transformations of integrable systems, called Bäcklund transformations. [5] The connection between those transformations and our ones is presently unclear. Thus, we have found it useful to call our transformations differently, and the results described below have prompted us to use for them the term 'canonical symmetry transformations'. Nevertheless, we would like to point out that the usual Bäcklund transformation are also canonical transformations (see, for example, Ref. 7).

\section{Nonlinear Schrödinger Equation}

As the first example we consider the canonical symmetry of the nonlinear Schrödinger equation. It is a nonlinear equation for a complex valued function $\psi(x, t)$ of the form

$$
i \dot{\psi}+\psi^{\prime \prime}-2 \kappa|\psi|^{2} \psi=0
$$

Here and below dot means the partial derivative over $t$ and prime - the partial derivative over $x$. The real parameter $\kappa$ is the coupling constant. We denote the complex conjugation by bar, so that $|\psi|^{2}=\bar{\psi} \psi$. Note, that we also have the complex conjugate equation

$$
i \dot{\bar{\psi}}-\bar{\psi}^{\prime \prime}+2 \kappa|\psi|^{2} \bar{\psi}=0
$$

Equations (2.1) and (2.2) are the Euler-Lagrange equations for the Lagrangian

$$
L=\int d x\left(\frac{i}{2}(\bar{\psi} \dot{\psi}-\dot{\bar{\psi}} \psi)-\left|\psi^{\prime}\right|^{2}-\kappa|\psi|^{4}\right) .
$$

We see that the action, corresponding to the Lagrangian (2.3), is already in the Hamiltonian form. Thus we can consider the space with the coordinates $\psi(x)$ and $\bar{\psi}(x)$ as the phase 
space of the system. The nonzero Poisson brackets in this case are

$$
\left\{\psi(x), \bar{\psi}\left(x^{\prime}\right)\right\}=-i \delta\left(x-x^{\prime}\right) .
$$

These Poisson brackets are generated by the symplectic 2 -form

$$
\Omega=i \int d x d \bar{\psi} \wedge d \psi
$$

For the Hamiltonian of the system we have the expression

$$
H=\int d x\left(\left|\psi^{\prime}\right|^{2}+\kappa|\psi|^{4}\right)
$$

It is easy to verify that this Hamiltonian with the Poisson brackets, defined by (2.4), leads to nonlinear Schrödinger equation (2.1), (2.2).

To define the canonical symmetry transformation we should construct a complex extension of the system. To this end we denote $q=\psi, r=\bar{\psi}$ and consider $q$ and $r$ as independent complex valued functions, satisfying the following system of equations

$$
i \dot{q}+q^{\prime \prime}-2 \kappa r q^{2}=0, \quad i \dot{r}-r^{\prime \prime}+2 \kappa q r^{2}=0
$$

These equations can be derived from the action with the density of the Lagrangian

$$
\mathcal{L}=\frac{i}{2}(r \dot{q}-\dot{r} q)-q^{\prime} r^{\prime}-\kappa q^{2} r^{2}
$$

This density of the Lagrangian is complex valued and not appropriate to formulate the Hamiltonian description of the system. We will use a real Lagrangian

$$
L=\frac{1}{2} \int d x(\mathcal{L}+\overline{\mathcal{L}})
$$

which leads to the same equations.

Proceeding as above, we get the phase space with the coordinates $q, r$ and $\bar{q}$, $\bar{r}$. The symplectic 2 -form in this case is of the form

$$
\Omega=\frac{i}{2} \int d x(d r \wedge d q-d \bar{r} \wedge d \bar{q})
$$

and for the nonzero Poisson brackets we have the expressions

$$
\left\{q(x), r\left(x^{\prime}\right)\right\}=-2 i \delta\left(x-x^{\prime}\right), \quad\left\{\bar{q}(x), \bar{r}\left(x^{\prime}\right)\right\}=2 i \delta\left(x-x^{\prime}\right) .
$$

The Hamiltonian of the system is

$$
H=\frac{1}{2} \int d x(\mathcal{H}+\overline{\mathcal{H}})
$$

where

$$
\mathcal{H}=r^{\prime} q^{\prime}+\kappa r^{2} q^{2}
$$


Below, considering complex extensions, we use Lagrangians and Hamiltonians of form (2.9) and (2.12). In such cases we call $\mathcal{L}$ and $\mathcal{H}$ the density of the Lagrangian and the Hamiltonian, respectively.

To get the initial nonlinear Schrödinger system it is enough to perform the reduction to the surface, given by the equations

$$
r=\bar{q}, \quad \bar{r}=q .
$$

The canonical symmetry transformation in this case has the form[1], 3, 7]

$$
Q=\frac{1}{\kappa r}, \quad R=\kappa r^{2} q-r^{\prime \prime}+\frac{r^{2}}{r} .
$$

There are of course the corresponding formulae for the complex conjugated functions $\bar{Q}$ and $\bar{R}$. Usually we will not write such formulae, because they can be obtained simply by the complex conjugation. Note, that the surface, given by (2.14), is not invariant under transformation (2.15), and it is the reason for introducing a complex extension of the system.

We are going to show that transformation (2.15) is canonical. To do this it is convenient to use the method of generating functions (functionals). \& In our case this method is as follows.

The symplectic 2 -form $\Omega$, given by $(2.10)$ is exact and can be represented as

$$
\Omega=d \Theta,
$$

where

$$
\Theta=\frac{i}{2} \int d x(r d q-\bar{r} d \bar{q})
$$

Suppose that transformation from the variables $q, r$ and $\bar{q}, \bar{r}$ to the variables $Q, R$ and $\bar{Q}$, $\bar{R}$ is canonical. In this case we must have

$$
\frac{i}{2} \int d x(r d q-\bar{r} d \bar{q})=\frac{i}{2} \int d x(R d Q-\bar{R} d \bar{Q})+d F
$$

where $F$ is some real valued functional on the phase space. Suppose further that the transformation under consideration is such that the functions $q, Q$ and $\bar{q}$ and $\bar{Q}$ can be considered as coordinates on the phase space. Representing $d F$ in the form

$$
d F=\int d x\left(\frac{\delta F}{\delta q} d q+\frac{\delta F}{\delta Q} d Q+\frac{\delta F}{\delta \bar{q}} d \bar{q}+\frac{\delta F}{\delta \bar{Q}} d \bar{Q}\right)
$$

we get the following formulae for $r(x)$ and $R(x)$ :

$$
r=-2 i \frac{\delta F}{\delta q}, \quad R=2 i \frac{\delta F}{\delta Q} .
$$

It is also clear that for any real valued functional $F$ of the variables $q, Q$, and $\bar{q}, \bar{Q}$ formulae (2.20) define a canonical transformation, provided that they can be written as the relations, defining an invertible transformation. The functional $F$ is called the generating functional of the corresponding transformation. 
Let us return again to our example. From (2.15) we get

$$
r=\frac{1}{\kappa Q}, \quad R=\frac{1}{\kappa Q^{2}}\left(q+Q^{\prime \prime}-\frac{Q^{\prime 2}}{Q}\right) .
$$

It is clear that we can consider the functions $q, Q$ and $\bar{q}, \bar{Q}$ as coordinates on the phase space. Using now (2.21) in (2.20), we get the following expression for the generating functional:

$$
F=\frac{1}{2} \int d x(\mathcal{F}+\overline{\mathcal{F}})
$$

where

$$
\mathcal{F}=\frac{i}{\kappa}\left(\frac{q}{Q}+\frac{1}{2} \frac{Q^{\prime 2}}{Q^{2}}\right)
$$

Thus, we see that in this case the canonical symmetry is a canonical transformation.

Present now the formula that describes the behaviour of the density of the Hamiltonian $\mathcal{H}$ under the canonical symmetry transformation:

$$
\mathcal{H}(Q, R)=\mathcal{H}(q, r)+\left(-2 q r^{\prime}+\frac{r^{\prime \prime} r^{\prime}}{\kappa r^{2}}-\frac{2}{3} \frac{r^{\prime 3}}{\kappa r^{3}}\right)^{\prime} .
$$

From this relation we conclude, that under appropriate boundary conditions the Hamiltonian of the system is invariant under the canonical symmetry transformation.

The nonlinear Schrödinger equation is known to have an infinite set of local conservation laws. 99, 10 The same is true for the complex extension of the system, which is considered here. The densities of the lowest conservation laws are

$$
\begin{aligned}
& \mathcal{P}_{1}=r q, \quad \mathcal{P}_{2}=-i r q^{\prime}, \\
& \mathcal{P}_{3}=-r q^{\prime \prime}+\kappa r^{2} q^{2}, \\
& \mathcal{P}_{4}=i\left(r q^{\prime \prime \prime}-\kappa r q\left(r^{\prime} q+4 r q^{\prime}\right)\right) .
\end{aligned}
$$

Through direct calculation we can show that these densities are invariant under the canonical symmetry transformation up to the total derivatives, i. e.

$$
\mathcal{P}_{n}(Q, R)=\mathcal{P}_{n}(q, r)+\mathcal{D}_{n}^{\prime}(q, r),
$$

where

$$
\begin{aligned}
& \mathcal{D}_{1}=-\frac{1}{\kappa} \frac{r^{\prime}}{r}, \quad \mathcal{D}_{2}=i\left(r q-\frac{1}{2 \kappa} \frac{r^{\prime 2}}{r^{2}}\right) \\
& \mathcal{D}_{3}=r q^{\prime}-r^{\prime} q+\frac{1}{3 \kappa} \frac{r^{\prime 3}}{r^{3}} \\
& \mathcal{D}_{4}=i\left(-r q^{\prime \prime}+\frac{3}{2} \kappa r^{2} q^{2}+r^{\prime} q^{\prime}-\frac{r^{\prime 2} q}{r}+\frac{1}{4 \kappa} \frac{r^{\prime 4}}{r^{4}}\right) .
\end{aligned}
$$

It can be shown that all the densities of the local conservation laws obey this property. We shall give the proof of this fact in the subsequent paper. This property means, in particular, that the higher nonlinear Schrödinger equations are invariant under canonical symmetry transformation (2.15). 


\section{Modified Nonlinear Schrödinger Equation}

The modified nonlinear Schrödinger equation is the equation of the form 11

$$
i \dot{\psi}+\psi^{\prime \prime}-2 i \kappa|\psi|^{2} \psi^{\prime}=0
$$

The corresponding complex extension is the following system of equations:

$$
i \dot{q}+q^{\prime \prime}-2 i \kappa(r q) q^{\prime}=0, \quad i \dot{r}-r^{\prime \prime}-2 i \kappa(r q) r^{\prime}=0 .
$$

We consider this system as Euler-Lagrange equations, corresponding to the Lagrangian

$$
L=\frac{1}{2} \int d x(\mathcal{L}+\overline{\mathcal{L}})
$$

where

$$
\mathcal{L}=\frac{i}{2}(r \dot{q}-\dot{r} q)-r^{\prime} q^{\prime}-\frac{i \kappa}{2}\left(r^{2} q q^{\prime}-q^{2} r r^{\prime}\right) .
$$

From (3.4) we conclude that we can consider the space with the coordinates $q, r$ and $\bar{q}$, $\bar{r}$ as the phase space of the system, with the nonzero Poisson brackets (2.11). The density of the Hamiltonian is given by

$$
\mathcal{H}=q^{\prime} r^{\prime}+\frac{i \kappa}{2}\left(r^{2} q q^{\prime}-q^{2} r r^{\prime}\right)
$$

The canonical symmetry transformation for the case under consideration is 11, 3]

$$
Q=\frac{1}{\kappa r}, \quad R=\kappa r^{2} q+i\left(r^{\prime}-\frac{r^{\prime \prime} r}{r^{\prime}}\right) .
$$

Writing this transformation in the form

$$
r=\frac{1}{\kappa Q}, \quad R=\frac{1}{\kappa Q^{2}}\left[q+i\left(Q^{\prime}-\frac{Q^{\prime \prime} Q}{Q^{\prime}}\right)\right],
$$

and using (2.20), we can show that canonical symmetry transformation (3.6) is a canonical transformation with the generating functional, defined by the density

$$
\mathcal{F}=\frac{i}{\kappa}\left(\frac{q}{Q}+i \frac{Q^{\prime}}{Q} \ln Q^{\prime}\right)
$$

The formula that describes the behaviour of the density of the Hamiltonian (3.5) under canonical symmetry transformation (3.6) has the form

$$
\mathcal{H}(Q, R)=\mathcal{H}(q, r)+\frac{1}{2}\left[-3 r^{\prime} q-i \kappa r^{2} q^{2}-\frac{r^{\prime \prime} r q}{r^{\prime}}+\frac{i}{\kappa}\left(\frac{r^{\prime \prime}}{r}-\frac{1}{2} \frac{r^{\prime 2}}{r^{2}}+\frac{1}{2} \frac{r^{\prime \prime 2}}{r^{\prime 2}}\right)\right]^{\prime} .
$$




\section{Derivative Nonlinear Schrödinger Equation}

The derivative nonlinear Schrödinger equation has the form 12

$$
i \dot{\psi}+\psi^{\prime \prime}-2 i \kappa\left(|\psi|^{2} \psi\right)^{\prime}=0
$$

Thus, we have the following complex extension

$$
i \dot{q}+q^{\prime \prime}-2 i \kappa\left(r q^{2}\right)^{\prime}=0, \quad i \dot{r}-r^{\prime \prime}-2 i \kappa\left(r^{2} q\right)^{\prime}=0 .
$$

Let us give at once the Hamiltonian formulation for this system. The phase space is again the space with the coordinates $q, r$ and $\bar{q}, \bar{r}$, but the nonzero Poisson brackets have now the form

$$
\left\{q(x), r\left(x^{\prime}\right)\right\}=2 \delta^{\prime}\left(x-x^{\prime}\right), \quad\left\{\bar{q}(x), \bar{r}\left(x^{\prime}\right)\right\}=2 \delta^{\prime}\left(x-x^{\prime}\right),
$$

and the density of the Hamiltonian is

$$
\mathcal{H}=\frac{i}{2}\left(r q^{\prime}-r^{\prime} q\right)+\kappa r^{2} q^{2}
$$

Poisson brackets (4.3) are generated by the symplectic 2-form

$$
\Omega=\frac{1}{2} \int d x\left(\partial^{-1} d r \wedge d q+\partial^{-1} d \bar{r} \wedge d \bar{q}\right)
$$

where the operator $\partial^{-1}$ acts on a function $f(x)$, for example, according to the rule

$$
\partial^{-1} f(x)=\frac{1}{2}\left(\int_{-\infty}^{x} d x^{\prime} f\left(x^{\prime}\right)-\int_{x}^{\infty} d x^{\prime} f\left(x^{\prime}\right)\right)
$$

The 2 -form $\Omega$ is exact and can be represented as

$$
\Omega=d \Theta,
$$

where

$$
\Theta=\frac{1}{2} \int d x\left(\partial^{-1} r d q+\partial^{-1} \bar{r} d \bar{q}\right)
$$

The canonical symmetry transformation for the derivative nonlinear Schrödinger equation is of the form [1, 3]

$$
Q=r, \quad R=q-\frac{i}{\kappa} \frac{r^{\prime}}{r^{2}}
$$

As we have a noncanonical symplectic 2-form, in this case we can not use formulae (2.20). Using the method, considered in section 2, we get that now the corresponding formulae are

$$
r=2\left(\frac{\delta F}{\delta q}\right)^{\prime}, \quad R=-2\left(\frac{\delta F}{\delta Q}\right)^{\prime} .
$$

Writing now the canonical symmetry transformation as

$$
r=Q, \quad R=q-\frac{i}{\kappa} \frac{Q^{\prime}}{Q^{2}},
$$


and using (4.10), we see that canonical symmetry transformation (4.9) is canonical and the density of the generating functional is

$$
\mathcal{F}=q \partial^{-1} Q-\frac{i}{\kappa} \ln Q
$$

The behaviour of the quantity $\mathcal{H}$ under the canonical symmetry transformation is described by the formula

$$
\mathcal{H}(Q, R)=\mathcal{H}(q, r)+\left(-i q r-\frac{1}{2 \kappa} \frac{r^{\prime}}{r}\right)^{\prime}
$$

\section{$5 \quad$ Heisenberg Model}

The phase space of the Heisenberg model is formed by real vector functions $\vec{S}(x)=$ $\left(S_{1}(x), S_{2}(x), S_{3}(x)\right)$, subjected to the condition

$$
\vec{S}^{2}(x)=1
$$

The equations of motion have the form

$$
\dot{\vec{S}}=\vec{S} \times \vec{S}^{\prime \prime}
$$

The Poisson brackets are given by

$$
\left\{S_{i}(x), S_{j}\left(x^{\prime}\right)\right\}=-\varepsilon_{i j k} S_{k}(x) \delta\left(x-x^{\prime}\right) .
$$

It is easy to show that equations (5.1) can be written as the Hamilton equations

$$
\dot{\vec{S}}=\{\vec{S}, H\}
$$

with the Hamiltonian

$$
H=\frac{1}{2} \int d x\left(\vec{S}^{\prime}\right)^{2}
$$

Introduce the stereographic coordinates

$$
\psi=\frac{S_{1}+i S_{2}}{1+S_{3}}, \quad \bar{\psi}=\frac{S_{1}-i S_{2}}{1+S_{3}} .
$$

For the Poisson brackets of $\psi$ and $\bar{\psi}$ we find the expressions

$$
\begin{gathered}
\left\{\psi(x), \psi\left(x^{\prime}\right)\right\}=0, \quad\left\{\bar{\psi}(x), \bar{\psi}\left(x^{\prime}\right)\right\}=0, \\
\left\{\psi(x), \bar{\psi}\left(x^{\prime}\right)\right\}=-\frac{i}{2}\left(1+|\psi|^{2}(x)\right)^{2} \delta\left(x-x^{\prime}\right),
\end{gathered}
$$

and the Hamiltonian in terms of the new variables becomes

$$
H=\int d x \frac{2\left|\psi^{\prime}\right|^{2}}{\left(1+|\psi|^{2}\right)^{2}} .
$$


Using these relations, we see that the equations of motion can be written in the form

$$
i \dot{\psi}+\psi^{\prime \prime}-\frac{2 \bar{\psi} \psi^{\prime 2}}{1+|\psi|^{2}}=0 .
$$

Consider as a complex extension the following system of equations

$$
i \dot{q}+q^{\prime \prime}-\frac{2 r q^{\prime 2}}{1+r q}=0, \quad i \dot{r}-r^{\prime \prime}+\frac{2 q r^{2}}{1+r q}=0 .
$$

In fact, we can get this complex extension, if from the beginning suppose that $\vec{S}$ is a complex valued vector function. The nonzero Poisson brackets in this case are

$$
\begin{gathered}
\left\{q(x), r\left(x^{\prime}\right)\right\}=-i(1+r q(x))^{2} \delta\left(x-x^{\prime}\right), \\
\left\{\bar{q}(x), \bar{r}\left(x^{\prime}\right)\right\}=i(1+\bar{r} \bar{q}(x))^{2} \delta\left(x-x^{\prime}\right),
\end{gathered}
$$

and the density of the Hamiltonian is given by

$$
\mathcal{H}=\frac{2 r^{\prime} q^{\prime}}{(1+r q)^{2}} .
$$

Poisson brackets (5.12), (5.13) are generated by the symplectic 2 -form

$$
\Omega=i \int d x\left(\frac{d r \wedge d q}{(1+r q)^{2}}-\frac{d \bar{r} \wedge d \bar{q}}{(1+\bar{r} \bar{q})^{2}}\right),
$$

which can be represented as

$$
\Omega=d \Theta,
$$

where

$$
\Theta=-i \int d x\left(\frac{d q}{q(1+r q)}-\frac{d \bar{q}}{\bar{q}(1+\bar{r} \bar{q})}\right) .
$$

Consider now a canonical transformation from the variables $q, r$ and $\bar{r}, \bar{q}$, to the variables $Q, R$ and $\bar{Q}, \bar{R}$, and suppose that the variables $q, Q$ and $\bar{q}, \bar{Q}$ can be considered as coordinates on the phase space. Using (5.17), it can be directly shown that in this case we can write

$$
\frac{1}{q(1+r q)}=i \frac{\delta F}{\delta q}, \quad \frac{1}{Q(1+R Q)}=-i \frac{\delta F}{\delta Q},
$$

where $F$ is a functional of $q, Q$ and $\bar{q}, \bar{Q}$.

The canonical symmetry transformation in this case has the form

$$
Q=\frac{1}{r}, \quad \frac{1}{1+R Q}-\frac{1}{1+r q}=\frac{r r^{\prime \prime}-r^{\prime 2}}{r^{\prime 2}} .
$$

From (5.18) and (5.19) we find that the density of the generating functional has the form

$$
\mathcal{F}=-2 i \ln \frac{q Q^{\prime}}{Q(Q+q)} .
$$

Thus we see that canonical symmetry transformation (5.19) is a canonical transformation.

For the transformation of the density of the Hamiltonian we have the formula

$$
\mathcal{H}(Q, R)=\mathcal{H}(q, r)+\left(-\frac{4 r^{\prime} q}{1+r q}+\frac{2 r^{\prime \prime}}{r^{\prime}}\right)^{\prime} .
$$




\section{Landau-Lifshits Model}

This model is a generalization of the Heisenberg model. The equations of motion in this case are

$$
\dot{\vec{S}}=\vec{S} \times \vec{S}^{\prime \prime}+\vec{S} \times J \vec{S},
$$

where $J$ is a constant symmetric matrix, that can be chosen as a diagonal one: $J=$ $\operatorname{diag}\left(J_{1}, J_{2}, J_{3}\right)$. Poisson brackets (5.3) lead to equations (6.1) if we define the Hamiltonian as

$$
H=\frac{1}{2} \int d x\left(\vec{S}^{\prime 2}-\vec{S} J \vec{S}\right) .
$$

Using the stereographic coordinates, given by (5.6), we get the following expression for the Hamiltonian:

$$
H=\int d x\left(\frac{2\left(\left|\psi^{\prime}\right|^{2}+\alpha\left(\psi^{2}+\bar{\psi}^{2}\right)-\gamma|\psi|^{2}\right)}{\left(1+|\psi|^{2}\right)^{2}}-\beta\right),
$$

where

$$
\alpha=\frac{J_{2}-J_{1}}{4}, \quad \beta=\frac{J_{3}}{2}, \quad \gamma=\frac{J_{1}+J_{2}}{2}-J_{3} .
$$

It is clear that the constant term in the density of the Hamiltonian can be excluded from the consideration.

The Poisson brackets for $\psi$ and $\bar{\psi}$ are given by (5.7) and (5.8), and the Hamilton equation has the form

$$
i \dot{\psi}+\psi^{\prime \prime}-\frac{2 \bar{\psi} \psi^{\prime 2}-2 \alpha \psi^{3}+\gamma \bar{\psi} \psi^{2}-\gamma \psi+2 \alpha \bar{\psi}}{1+|\psi|^{2}}=0
$$

Denoting

$$
\rho(x)=\alpha x^{4}+\gamma x^{2}+\alpha,
$$

we write equation (6.5) in a more compact form

$$
i \dot{\psi}+\psi^{\prime \prime}-\frac{2 \bar{\psi}\left(\psi^{\prime 2}+\rho(\psi)\right)}{1+|\psi|^{2}}+\frac{1}{2} \rho^{\prime}(\psi)=0
$$

Construct now a complex extension of the system. The corresponding equations are

$$
\begin{aligned}
& i \dot{q}+q^{\prime \prime}-\frac{2 r\left(q^{2}+\rho(q)\right)}{1+r q}+\frac{1}{2} \rho^{\prime}(q)=0 \\
& i \dot{r}-r^{\prime \prime}+\frac{2 q\left(r^{\prime 2}+\rho(r)\right)}{1+r q}-\frac{1}{2} \rho^{\prime}(r)=0 .
\end{aligned}
$$

These equations are Hamilton equations for the Hamiltonian defined by the density

$$
\mathcal{H}=\frac{2\left(r^{\prime} q^{\prime}+\alpha\left(r^{2}+q^{2}\right)-\gamma r q\right)}{(1+r q)^{2}} .
$$

The canonical symmetry transformation for the Landau-Lifshits model is

$$
Q=\frac{1}{r}, \quad \frac{1}{1+R Q}-\frac{1}{1+r q}=\frac{r r^{\prime \prime}-r^{\prime 2}+\alpha r^{4}-\alpha}{r^{\prime 2}+\rho(r)} .
$$


It can be shown that this transformation is canonical and the density of the generating functional has the form

$$
\mathcal{F}=-i \ln \frac{q^{2}\left(Q^{\prime 2}+\rho(Q)\right)}{Q^{2}(q+Q)^{2}}+2 i \frac{Q^{\prime}}{\rho^{1 / 2}(Q)} \arctan \frac{Q^{\prime}}{\rho^{1 / 2}(Q)} .
$$

Note, that without any loose of generality we can consider $\alpha$ as a positive constant. In this case the function $\rho(x)$ is positively definite.

The behaviour of the density of the Hamiltonian under the canonical symmetry transformation is described by the formula

$$
\mathcal{H}(Q, R)=\mathcal{H}(q, r)+\left(-\frac{4 r^{\prime} q}{1+r q}+\frac{2 r^{\prime \prime} r^{\prime}+r^{\prime} \rho^{\prime}(r)}{r^{\prime 2}+\rho(r)}\right)^{\prime} .
$$

\section{Principal Chiral Field}

The principal chiral field is a function on the space-time taking values in a Lie group $G$. We consider here the case of the two-dimensional Minkowski space-time, and use from the beginning a complex extension of the system, supposing that $G$ is a complex matrix Lie group. The equations of motion for the principal chiral field $g(t, x)$ have the form

$$
\left(g^{-1} g_{, t}\right)_{, t}-\left(g^{-1} g_{, x}\right)_{, x}=0
$$

From these equations we see that there exists a function $\varphi(t, x)$ taking values in the corresponding Lie algebra $\mathcal{G}$ such that

$$
g^{-1} g_{, t}=\varphi_{, x}, \quad g^{-1} g_{, x}=\varphi_{, t}
$$

If we take some function $\varphi$ and solve equations (7.2) we get a solution of equations (7.1). Note, that the integrability conditions of equations (7.2), considered as equations for the function $g$, has the form

$$
\varphi_{, t t}-\varphi_{, x x}=\left[\varphi_{, t}, \varphi_{, x}\right] .
$$

Hence, only those functions $\varphi$, that satisfy equations (7.3), give solutions of equations (7.1). It is clear that any solution of equations (7.1) can be obtained in such a way. Thus, the integration problem of equations (7.1) is reduced to the integration problem of equations (7.3).

Equations (7.3) are Lagrange equations for the action defined by the density of the Lagrangian

$$
\mathcal{L}=\operatorname{tr}\left(\frac{1}{2}\left(\varphi_{, t}^{2}-\varphi_{, x}^{2}\right)+\frac{1}{3} \varphi\left[\varphi_{, t}, \varphi_{, x}\right]\right) .
$$

We restrict ourself to the case $G=\mathrm{SL}(2, \mathbf{C}), \mathcal{G}=\mathrm{sl}(2, \mathbf{C})=A_{1}$. Recall, that the group $\mathrm{SL}(2, \mathbf{C})$ is the group of complex $2 \times 2$-matrices with the determinant equal to one. The Lie algebra $\operatorname{sl}(2, \mathbf{C})$ of the group $\operatorname{SL}(2, \mathbf{C})$ consists of all complex traceless $2 \times 2$-matrices. The matrices

$$
H=\left(\begin{array}{rr}
1 & 0 \\
0 & -1
\end{array}\right), \quad X_{+}=\left(\begin{array}{ll}
0 & 1 \\
0 & 0
\end{array}\right), \quad X_{-}=\left(\begin{array}{ll}
0 & 0 \\
1 & 0
\end{array}\right)
$$


form a basis in the Lie algebra $\mathrm{sl}(2, \mathbf{C})$. The commutation relations for these matrices have the form

$$
\left[H, X_{+}\right]=2 X_{+}, \quad\left[H, X_{-}\right]=-2 X_{-}, \quad\left[X_{+}, X_{-}\right]=H .
$$

Represent the field $\varphi$ in the form

$$
\varphi=\varphi^{-} X_{-}+\varphi^{0} H+\varphi^{+} X_{+}=\left(\begin{array}{cc}
\varphi^{0} & \varphi^{+} \\
\varphi^{-} & -\varphi^{0}
\end{array}\right) .
$$

Using this representation, we get from (7.3) the following system of equations for $\varphi^{-}, \varphi^{0}$ and $\varphi^{+}$:

$$
\begin{aligned}
& \varphi_{, t t}^{-}-\varphi_{, x x}^{-}=-2\left(\varphi_{, t}^{0} \varphi_{, x}^{-}-\varphi_{, t}^{-} \varphi_{, x}^{0}\right), \\
& \varphi_{, t t}^{0}-\varphi_{, x x}^{0}=\varphi_{, t}^{+} \varphi_{, x}^{-}-\varphi_{, t}^{-} \varphi_{, x}^{+}, \\
& \varphi_{, t t}^{+}-\varphi_{, x x}^{+}=2\left(\varphi_{, t}^{0} \varphi_{, x}^{+}-\varphi_{, t}^{+} \varphi_{, x}^{0}\right) .
\end{aligned}
$$

The density of the Lagrangian can be written for this case in the form

$$
\begin{aligned}
\mathcal{L}=\left(\varphi_{, t}^{0}\right)^{2} & +\varphi_{, t}^{-} \varphi_{, t}^{+}-\left(\varphi_{, x}^{0}\right)^{2}-\varphi_{, x}^{-} \varphi_{, x}^{+}+\frac{2}{3}\left(\varphi^{0}\left(\varphi_{, t}^{+} \varphi_{, x}^{-}-\varphi_{, t}^{-} \varphi_{, x}^{+}\right)\right. \\
& \left.+\varphi^{-}\left(\varphi_{, t}^{0} \varphi_{, x}^{+}-\varphi_{, t}^{+} \varphi_{, x}^{0}\right)-\varphi^{+}\left(\varphi_{, t}^{0} \varphi_{, x}^{-}-\varphi_{, t}^{-} \varphi_{, x}^{0}\right)\right) .
\end{aligned}
$$

It is more convenient to use the density of the Lagrangian

$$
\mathcal{L}=\left(\varphi_{, t}^{0}\right)^{2}+\varphi_{, t}^{-} \varphi_{, t}^{+}-\left(\varphi_{, x}^{0}\right)^{2}-\varphi_{, x}^{-} \varphi_{, x}^{+}+2 \varphi^{-}\left(\varphi_{, t}^{0} \varphi_{, x}^{+}-\varphi_{, t}^{+} \varphi_{, x}^{0}\right),
$$

which differs from the one, given by (7.11), by divergency terms.

The canonical symmetry transformation for the case under consideration is determined from the relations[1], 3]:

$$
\begin{aligned}
& \Phi^{-}=-\frac{1}{\varphi^{-}}, \\
& \Phi_{, t}^{0}=\varphi_{, t}^{0}-\left(\varphi^{0}+\Phi^{0}\right) \frac{\varphi_{, t}^{-}}{\varphi^{-}}-\frac{\varphi_{, x}^{-}}{\varphi^{-}} \\
& \Phi_{, x}^{0}=\varphi_{, x}^{0}-\left(\varphi^{0}+\Phi^{0}\right) \frac{\varphi_{, x}^{-}}{\varphi^{-}}-\frac{\varphi_{, t}^{-}}{\varphi^{-}}, \\
& \Phi_{, t}^{+}=\varphi^{-2} \varphi_{, t}^{+}-\left(\varphi^{0}+\Phi^{0}\right)^{2} \varphi_{, t}^{-}-\varphi_{, t}^{-} \\
& \quad+2 \varphi^{-}\left(\varphi^{0}+\Phi^{0}\right) \varphi_{, t}^{0}-2\left(\varphi^{0}+\Phi^{0}\right) \varphi_{, x}^{-}+2 \varphi^{-} \varphi_{, x}^{0}, \\
& \Phi_{, x}^{+=} \varphi^{-2} \varphi_{, x}^{+}-\left(\varphi^{0}+\Phi^{0}\right)^{2} \varphi_{, x}^{-}-\varphi_{, x}^{-} \\
& \quad+2 \varphi^{-}\left(\varphi^{0}+\Phi^{0}\right) \varphi_{, x}^{0}-2\left(\varphi^{0}+\Phi^{0}\right) \varphi_{, t}^{-}+2 \varphi^{-} \varphi_{, t}^{0} .
\end{aligned}
$$

In contrast to the examples, considered above, the canonical symmetry transformation for the principal chiral field is given in an implicit form. To get the explicit relations we have to integrate relations (7.13)-(7.17). For example, from (7.15) we have

$$
\Phi^{0}=\partial^{-1} \frac{1}{\varphi^{-}}\left(\varphi_{, x}^{0} \varphi^{-}-\varphi^{0} \varphi_{, x}^{-}-\varphi_{, t}^{-}\right)
$$


where operator $\partial^{-1}$ is defined by (4.6).

From (7.12) we get for the density of the energy of the system the following expression:

$$
\mathcal{E}=\left(\varphi_{, t}^{0}\right)^{2}+\varphi_{, t}^{-} \varphi_{, t}^{+}+\left(\varphi_{, x}^{0}\right)^{2}+\varphi_{, x}^{-} \varphi_{, x}^{+} .
$$

The direct calculation shows that this density is invariant under the canonical symmetry transformation. Note, that it is enough here to use only nonintegrated relations (7.14)(7.17). From this invariance it follows that the density of the Hamiltonian of the system is invariant under the canonical symmetry transformation.

From (7.12) we see that the generalized momenta are given by

$$
\pi^{-}=\frac{1}{2} \varphi_{, t}^{+}, \quad \pi^{0}=\varphi_{, t}^{0}+\varphi^{-} \varphi_{, x}^{+}, \quad \pi^{+}=\frac{1}{2} \varphi_{, t}^{-}-\varphi^{-} \varphi_{, x}^{0} .
$$

Consider canonical symmetry transformation (7.13)-(7.17) as a transformation in the phase space of the system, that connects the coordinates $\varphi^{-}, \varphi^{0}, \varphi^{+}$and $\pi^{-}, \pi^{0}, \pi^{+}$with the new coordinates $\Phi^{-}, \Phi^{0}, \Phi^{+}$and $\Pi^{-}, \Pi^{0}, \Pi^{+}$. Let us show that this transformation is canonical. It appears that the quantities $\Phi^{-}, \Phi^{0}, \Phi^{+}$and $\pi^{-}, \pi^{0}, \pi^{+}$can be considered as coordinates on the phase space. To demonstrate this we write the relations

$$
\begin{aligned}
& \varphi^{-}=-\frac{1}{\Phi^{-}} \\
& \varphi^{0}=-\Phi^{0}+2 \Phi^{-} \partial^{-1} \pi^{+} \\
& \varphi^{+}=-\partial^{-1}\left(\Phi^{-2} \Phi_{, x}^{+}+\Phi_{, x}^{-}+2 \Phi^{-} \pi^{0}\right. \\
& \quad\left.\quad+4 \Phi_{, x}^{0} \Phi^{-2} \partial^{-1} \pi^{+}-4 \Phi_{, x}^{-} \Phi^{-2}\left(\partial^{-1} \pi^{+}\right)^{2}\right), \\
& \quad+\Phi^{-} \Phi_{, x}^{0}\left(\partial^{-1} \pi^{+}\right)^{2}+4 \Phi^{-2} \pi^{+}\left(\partial^{-1} \pi^{+}\right)^{2}, \\
& \Pi^{-}= \frac{\pi^{-}}{\Phi^{-2}}-\pi^{+}+2 \Phi^{-} \Phi_{, x}^{+} \partial^{-1} \pi^{+}+2 \pi^{0} \partial^{-1} \pi^{+} \\
& \Pi^{0}=-\pi^{0}-2\left(\Phi^{-2}\left(\partial^{-1} \pi^{+}\right)^{2}\right)_{, x}, \\
& \Pi^{+}=-\left(\Phi^{-2} \partial^{-1} \pi^{+}\right)_{, x} .
\end{aligned}
$$

Following the consideration of section 2, we conclude that if the transformation under consideration is canonical then we can represent $(7.21)-(7.26)$ in the form

$$
\begin{aligned}
& \varphi^{-}=\frac{\delta F}{\delta \pi^{-}}, \quad \varphi^{0}=\frac{\delta F}{\delta \pi^{0}}, \quad \varphi^{+}=\frac{\delta F}{\delta \pi^{+}}, \\
& \Pi^{-}=\frac{\delta F}{\delta \Phi^{-}}, \quad \Pi^{0}=\frac{\delta F}{\delta \Phi^{0}}, \quad \Pi^{+}=\frac{\delta F}{\delta \Phi^{+}},
\end{aligned}
$$

where $F$ is a functional of $\Phi^{-}, \Phi^{0}, \Phi^{+}$and $\pi^{-}, \pi^{0}, \pi^{+}$. And vice versa, if we can represent (7.21)-(7.26) in form (7.27), (7.28), then we are dealing with a canonical transformation. It is easy to get convinced, that if we choose $F$ as the functional, defined by the density

$$
\begin{aligned}
\mathcal{F}= & -\frac{2 \pi^{-}}{\Phi^{-}}-2 \Phi^{0} \pi^{0}-2 \Phi^{-} \pi^{+}+2 \Phi^{-2} \Phi_{, x}^{+} \partial^{-1} \pi^{+} \\
& +4 \Phi^{-} \pi^{0} \partial^{-1} \pi^{+}+4 \Phi^{-2} \Phi_{, x}^{0}\left(\partial^{-1} \pi^{+}\right)^{2}+\frac{8}{3} \Phi^{-3} \pi^{+}\left(\partial^{-1} \pi^{+}\right)^{2},
\end{aligned}
$$

then relations (7.21)-(7.26) will be satisfied. This ends the proof of the fact, that the canonical symmetry transformation for the principal chiral field is canonical. 


\section{Self-Dual Yang-Mills System}

In this section we consider the self-dual configurations of the Yang-Mills field in fourdimensional Euclidean space with coordinates $x^{\mu}, \mu=1,2,3,4$. We also use the notations $x^{1}=t, x^{2}=u, x^{3}=v$ and $x^{4}=w$. Consider the case, when the gauge group $G$ is a complex matrix Lie group. The gauge potential $a_{\mu}$ takes values in the corresponding Lie algebra $\mathcal{G}$. The field strength $f_{\mu \nu}$ is defined by

$$
f_{\mu \nu}=a_{\nu, \mu}-a_{\mu, \nu}+\left[a_{\mu}, a_{\nu}\right]
$$

The self-duality equations have the form

$$
f_{\mu \nu}=\frac{1}{2} \varepsilon_{\mu \nu \rho \sigma} f_{\rho \sigma}
$$

In fact, we have only three independent equations, that can be written as

$$
f_{t u}=f_{v w}, \quad f_{t v}=-f_{u w}, \quad f_{t w}=f_{u v} .
$$

Introduce four new complex variables defined by

$$
y=t+i u, \quad \bar{y}=t-i u, \quad z=v-i w, \quad \bar{z}=v+i w .
$$

The components of the field strength in the old and new coordinates are connected by the relations

$$
\begin{gathered}
f_{y \bar{y}}=\frac{i}{2} f_{t u}, \quad f_{z \bar{z}}=-\frac{i}{2} f_{v w}, \\
f_{y z}=\frac{1}{4}\left(f_{t v}+i f_{t w}+f_{u w}-i f_{u v}\right), \\
f_{\bar{y} \bar{z}}=\frac{1}{4}\left(f_{t v}-i f_{t w}+f_{u w}+i f_{u v}\right), \\
f_{y \bar{z}}=\frac{1}{4}\left(f_{t v}-i f_{t w}-f_{u w}-i f_{u v}\right), \\
f_{\bar{y} z}=\frac{1}{4}\left(f_{t v}+i f_{t w}-f_{u w}+i f_{u v}\right) .
\end{gathered}
$$

It is easy to check that in the new coordinates the self-duality equations reduce to

$$
\begin{gathered}
f_{y z}=0, \quad f_{\bar{y} \bar{z}}=0, \\
f_{y \bar{y}}+f_{z \bar{z}}=0 .
\end{gathered}
$$

From (8.8) it follows that we can find two matrix functions $h$ and $\bar{h}$ such that

$$
\begin{array}{ll}
a_{y}=h^{-1} h_{, y}, & a_{z}=h^{-1} h_{, z}, \\
a_{\bar{y}}=\bar{h}^{-1} \bar{h}_{, \bar{y}}, & a_{\bar{z}}=\bar{h}^{-1} \bar{h}_{, \bar{z}} .
\end{array}
$$

Using (8.10) and (8.11), we get for the field strength components $f_{y \bar{y}}$ and $f_{z \bar{z}}$ the representation

$$
f_{y \bar{y}}=-h^{-1}\left(g^{-1} g_{, \bar{y}}\right)_{, y} h, \quad f_{z \bar{z}}=-h^{-1}\left(g^{-1} g_{, \bar{z}}\right)_{, z} h,
$$

where $g=\bar{h} h^{-1}$. Thus, self-duality equation (8.9) takes the form

$$
\left(g^{-1} g_{, \bar{y}}\right)_{, y}+\left(g^{-1} g_{, \bar{z}}\right)_{, z}=0 .
$$


This is the so-called Yang equation. 13, 14

From (8.13) we conclude that there exists a function $\varphi$, taking values in the Lie algebra $\mathcal{G}$, such that

$$
g^{-1} g_{, \bar{y}}=\varphi_{, z}, \quad g^{-1} g_{, \bar{z}}=-\varphi_{, y} .
$$

If we chose some function $\varphi$, and solve the system of equations (8.14) we get a solution of the Yang equation, and any solution of this equation can be obtained in such a way. Note, that not every function $\varphi$ can be used in (8.14). The integrability conditions of this system of equation have the form

$$
\varphi_{, y \bar{y}}+\varphi_{, z \bar{z}}=\left[\varphi_{, y}, \varphi_{, z}\right],
$$

and if we wish to get a solution of the Yang equation we must choose the function $\varphi$, satisfying equation (8.15). Thus we reduce the problem of integration of the Yang equation to the integration of equation (8.15).

Equations (8.15) can be obtained from the action

$$
S=\frac{1}{2} \int d^{4} x(\mathcal{L}+\overline{\mathcal{L}}),
$$

where

$$
\mathcal{L}=2 \operatorname{tr}\left(\varphi_{, y} \varphi_{, \bar{y}}+\varphi_{, z} \varphi_{, \bar{z}}+\frac{2}{3} \varphi\left[\varphi_{, y}, \varphi_{, z}\right]\right) .
$$

The existence of this simple Lagrangian density is the reason for using equations (8.15) instead of the Yang equation.

Consider the case of the Lie algebra $\operatorname{sl}(2, \mathbf{C})=A_{1}$. In this case we present $\varphi$ in form (7.7) and get the following system of equations

$$
\begin{aligned}
& \varphi_{, y \bar{y}}^{-}+\varphi_{, z \bar{z}}^{-}=-2\left(\varphi_{, y}^{0} \varphi_{, z}^{-}-\varphi_{, y}^{-} \varphi_{, z}^{0}\right), \\
& \varphi_{, y \bar{y}}^{0}+\varphi_{, z \bar{z}}^{0}=\varphi_{, y}^{+} \varphi_{, z}^{-}-\varphi_{, y}^{-} \varphi_{, z}^{+}, \\
& \varphi_{, y \bar{y}}^{+}+\varphi_{, z \bar{z}}^{+}=2\left(\varphi_{, y}^{0} \varphi_{, z}^{+}-\varphi_{, y}^{+} \varphi_{, z}^{0}\right)
\end{aligned}
$$

The density of the Lagrangian up to divergency terms is

$$
\begin{aligned}
\mathcal{L}=4\left(\varphi_{, \bar{y}}^{0} \varphi_{, y}^{0}+\varphi_{, \bar{z}}^{0} \varphi_{, z}^{0}\right)+2 \varphi_{, \bar{y}}^{+} \varphi_{, y}^{-}+2 \varphi_{, \bar{y}}^{-} \varphi_{, y}^{+} & \\
& +2 \varphi_{, \bar{z}}^{+} \varphi_{, z}^{-}+2 \varphi_{, \bar{z}}^{-} \varphi_{, z}^{+}+8 \varphi^{-}\left(\varphi_{, y}^{0} \varphi_{, z}^{+}-\varphi_{, y}^{+} \varphi_{, z}^{0}\right) .
\end{aligned}
$$

The canonical symmetry transformation for this system has the form [2, 3]

$$
\begin{aligned}
\Phi^{-} & =-\frac{1}{\varphi^{-}}, \\
\Phi_{, y}^{0} & =\varphi_{, y}^{0}-\left(\varphi^{0}+\Phi^{0}\right) \frac{\varphi_{, y}^{-}}{\varphi^{-}}+\frac{\varphi_{, \bar{z}}^{-}}{\varphi^{-}}, \\
\Phi_{, z}^{0} & =\varphi_{, z}^{0}-\left(\varphi^{0}+\Phi^{0}\right) \frac{\varphi_{, z}^{-}}{\varphi^{-}}-\frac{\varphi_{, \bar{y}}^{-}}{\varphi^{-}}, \\
\Phi_{, y}^{+} & =\left(\varphi^{-}\right)^{2} \varphi_{, y}^{+}+\varphi^{-}\left[\left(\varphi^{0}+\Phi^{0}\right)\left(\varphi^{0}+\Phi^{0}\right)_{, y}-\left(\varphi^{0}+\Phi^{0}\right)_{, \bar{z}}\right], \\
\Phi_{, z}^{+} & =\left(\varphi^{-}\right)^{2} \varphi_{, z}^{+}+\varphi^{-}\left[\left(\varphi^{0}+\Phi^{0}\right)\left(\varphi^{0}+\Phi^{0}\right)_{, z}+\left(\varphi^{0}+\Phi^{0}\right)_{, \bar{y}}\right] .
\end{aligned}
$$


As in the case of the principal chiral field, the canonical symmetry transformation is given in an implicit form and require integration.

Using the relations

$$
\frac{\partial}{\partial y}=\frac{1}{2}\left(\frac{\partial}{\partial t}-i \frac{\partial}{\partial u}\right), \quad \frac{\partial}{\partial \bar{y}}=\frac{1}{2}\left(\frac{\partial}{\partial t}+i \frac{\partial}{\partial u}\right),
$$

we can rewrite the density of the Lagrangian in the form

$$
\begin{aligned}
\mathcal{L}= & \left(\varphi_{, t}^{0}\right)^{2}+\left(\varphi_{, u}^{0}\right)^{2}+\varphi_{, t}^{+} \varphi_{, t}^{-}+\varphi_{, u}^{+} \varphi_{, u}^{-}+4 \varphi_{, z}^{0} \varphi_{, \bar{z}}^{0}+2 \varphi_{, z}^{+} \varphi_{, z}^{-} \\
& +2 \varphi_{, \bar{z}}^{-} \varphi_{, z}^{+}+4 \varphi^{-}\left(\varphi_{, t}^{0} \varphi_{, z}^{+}-\varphi_{, t}^{+} \varphi_{, z}^{0}-i \varphi_{, u}^{0} \varphi_{, z}^{+}+i \varphi_{, u}^{+} \varphi_{, z}^{0}\right) .
\end{aligned}
$$

From this formula we get the following expressions for the generalized momenta:

$$
\pi^{-}=\frac{1}{2} \varphi_{, t}^{+}, \quad \pi^{0}=\varphi_{, t}^{0}+2 \varphi^{-} \varphi_{, z}^{+}, \quad \pi^{+}=\frac{1}{2} \varphi_{, t}^{-}-2 \varphi^{-} \varphi_{, z}^{0} .
$$

It is convenient for our purposes to use instead of the canonical coordinates $\varphi^{-}, \varphi^{0}, \varphi^{+}$and $\pi^{-}, \pi^{0}, \pi^{+}$another set of the canonical coordinates $\varphi^{-}, \varphi^{0}, \varphi^{+}$and $\tilde{\pi}^{-}, \pi^{0}, \tilde{\pi}^{+}$, where

$$
\tilde{\pi}^{-}=\pi^{-}-\frac{i}{2} \varphi_{, u}^{+}, \quad \tilde{\pi}^{+}=\pi^{+}+\frac{i}{2} \varphi_{, u}^{-}
$$

To show that the Hamiltonian version of canonical symmetry transformation (8.22)-(8.26) is a canonical transformation, we look for the generating functional of the type considered in the previous section. In other words, we suppose first that the quantities $\Phi^{-}, \Phi^{0}, \Phi^{+}$and $\tilde{\pi}^{-}, \pi^{0}, \tilde{\pi}^{+}$can be considered as coordinates on the phase space. It is really so, because we can rewrite relations (8.22)-(8.26) as the relations, connecting $\varphi^{-}, \varphi^{0}, \varphi^{+}$and $\widetilde{\Pi}^{-}, \Pi^{0}, \widetilde{\Pi}^{+}$ with $\Phi^{-}, \Phi^{0}, \Phi^{+}$and $\widetilde{\pi}^{-}, \pi^{0}, \widetilde{\pi}^{+}$:

$$
\begin{aligned}
\varphi^{-}= & -\frac{1}{\Phi^{-}} \\
\varphi^{0}= & -\Phi^{0}+\Phi^{-} \partial_{, z}^{-1} \widetilde{\pi}^{+} \\
\varphi^{+}= & \partial_{z}^{-1}\left(-\Phi^{-2} \Phi_{, z}^{+}+\Phi^{-2} \Phi_{, z}^{-}\left(\partial_{z}^{-1} \widetilde{\pi}^{+}\right)^{2}-\Phi^{-} \pi^{0}\right. \\
& \left.-2 \Phi^{-2} \Phi_{, z}^{0} \partial_{z}^{-1} \widetilde{\pi}^{+}+i \Phi^{-} \Phi_{, u}^{-} \partial_{z}^{-1} \widetilde{\pi}^{+}+\Phi_{, \bar{z}}^{-}-i \Phi^{-} \Phi_{, u}^{0}\right) \\
\widetilde{\Pi}^{-}= & \frac{\widetilde{\pi}^{-}}{\Phi^{-2}}+\pi^{0} \partial_{z}^{-1} \widetilde{\pi}^{+}+\Phi^{-2} \widetilde{\pi}^{+}\left(\partial_{z}^{-1} \widetilde{\pi}^{+}\right)^{2}+2 \Phi^{-} \Phi_{, z}^{0}\left(\partial^{-1} \widetilde{\pi}^{+}\right)^{2} \\
& +i \Phi^{-} \partial_{z}^{-1} \widetilde{\pi}_{, u}^{+} \partial_{z}^{-1} \widetilde{\pi}^{+}+2 \Phi^{-} \Phi_{, z}^{+} \partial_{z}^{-1} \widetilde{\pi}^{+}+i \Phi_{, u}^{0} \partial_{z}^{-1} \widetilde{\pi}^{+}+\partial^{-1} \pi_{, \bar{z}}^{+} \\
\Pi^{0}= & -\pi^{0}-\left(\Phi^{-2}\left(\partial_{z}^{-1} \widetilde{\pi}^{+}\right)^{2}\right)_{, z}-i\left(\Phi^{-} \partial_{z}^{-1} \widetilde{\pi}^{+}\right)_{, u}, \\
\widetilde{\Pi}^{+}= & -\left(\Phi^{-2} \partial_{z}^{-1} \widetilde{\pi}^{+}\right)_{, z} .
\end{aligned}
$$

Then we look for the functional $F$ of the variables $\Phi^{-}, \Phi^{0}, \Phi^{+}$and $\tilde{\pi}^{-}, \pi^{0}, \tilde{\pi}^{+}$, such that

$$
\begin{aligned}
& \varphi^{-}=\frac{\delta F}{\delta \widetilde{\pi}^{-}}, \quad \varphi^{0}=\frac{\delta F}{\delta \pi^{0}}, \quad \varphi^{+}=\frac{\delta F}{\delta \widetilde{\pi}^{+}}, \\
& \widetilde{\Pi}^{-}=\frac{\delta F}{\delta \Phi^{-}}, \quad \Pi^{0}=\frac{\delta F}{\delta \Phi^{0}}, \quad \widetilde{\Pi}^{+}=\frac{\delta F}{\delta \Phi^{+}} .
\end{aligned}
$$


It is easy to show that the functional $F$ does exist and its density has the form

$$
\begin{aligned}
\mathcal{F}= & -\frac{2 \widetilde{\pi}^{-}}{\Phi^{-}}-2 \Phi^{0} \pi^{0}+2 \Phi^{-} \partial_{z}^{-1} \widetilde{\pi}_{, \bar{z}}^{+} \\
& +2 \Phi^{-2} \Phi_{, z}^{+} \partial_{z}^{-1} \widetilde{\pi}^{+}+2 \Phi^{-} \pi^{0} \partial^{-1} \widetilde{\pi}^{+}+2 \Phi^{-2} \Phi_{, z}^{0}\left(\partial^{-1} \widetilde{\pi}^{+}\right)^{2} \\
& +\frac{2}{3} \Phi^{-3} \widetilde{\pi}^{+}\left(\partial_{z}^{-1} \widetilde{\pi}^{+}\right)^{2}+2 i \Phi^{-} \Phi_{, u}^{0} \partial_{z}^{-1} \widetilde{\pi}^{+}+i \Phi^{-2} \partial_{z}^{-1} \widetilde{\pi}_{, u}^{+} \partial_{z}^{-1} \widetilde{\pi}^{+}
\end{aligned}
$$

Thus, we see that we again have a canonical transformation.

\section{Conclusion}

The main result of the present paper, from our point of view, is not the concrete formulae, but the conclusions we can derive from them.

We believe that the integrability of a system is closely related to the existence of the corresponding canonical symmetry transformation. Moreover, all the main properties of the system can be derived from the properties of this transformation. The fact that for all considered cases, and for many others, which were not included in the present paper, the canonical symmetry transformations are canonical, is undoubtedly very important. But we do not know yet the direct connection of this fact with the integrability property.

It is known, that the integrability property is connected to the existence of a nontrivial algebra of internal symmetries. It is possible that this algebra is hidden in the corresponding canonical symmetry transformation.

\section{References}

[1] A. N. Leznov, in Proceedings of the First International A. D. Sakharov Conference on Physics (World Scientific, Singapore, 1991).

[2] A. N. Leznov, "Bäcklund Transformation of Self-Dual Yang-Mills Field for an Arbitrary Semisimple Gauge Algebra", IHEP preprint 91-145 (Protvino, 1991).

[3] A. N. Leznov, "Bäcklund Transformation for Integrable Systems", IHEP preprint 92-87 (Protvino, 1992).

[4] A. N. Leznov and M. V. Saveliev, Acta Appl. Math. 16, 1 (1989).

[5] Bäcklund Transformations, Lecture Notes in Mathematics, Vol. 515, edited by A. Dold and B. Eckmann (Springer, Berlin, 1976).

[6] H. Flaschka and D. W. McLaughlin, Ref. 6, pp. 253-295.

[7] A. B. Shabat and R. I. Yamilov, Leningrad Math. J. 2, 377 (1991).

[8] H. Goldstein, Classical Mechanics (Addison-Wesley, Cambridge, 1950).

[9] V. E. Zakharov and A. B. Shabat, Zh. Eksp. Teor. Fiz. 61, 118 (1971). 
[10] L. D. Faddeev and L. A. Takhtajan, Hamiltonian Methods in the Theory of Solitons (Springer, Berlin-Heidelberg-New York, 1987).

[11] H. H. Chen, Y. C. Lee and C. S. Lin, Phys. Scr. 20, 490 (1979).

[12] D. J. Kaup and A. C. Newell, J. Math. Phys. 19, 798 (1978).

[13] C. N. Yang, Phys. Rev. Lett. 38, 1377 (1977).

[14] Y. Brihaye, D. B. Fairlie, Y. Nuyts and R. G. Yates, J. Math. Phys. 19, 2528 (1978).

Received October 23, 1992. 